\title{
On fault tolerant control structures incorporating fault estimation
}

\author{
DUŠAN KROKAVEC, ANNA FILASOVÁ and PAVOL LIŠČINSKÝ
}

\begin{abstract}
The paper provides the minimal necessary modifications of linear matrix inequality conditions for the mixed $\mathrm{H}_{2} / \mathrm{H}_{\infty}$ control design as well as for the augmented observer-based fault estimation to be mutually compatible in joint design of integrated fault estimation and fault tolerant control. To be possible, within this integration, to design the controller which guarantees a pre-specified $\mathrm{H}_{\infty}$ norm disturbance attenuation level, the design conditions has to be regularized using the $\mathrm{H}_{2}$ performance index and, moreover, augmented fault observer must be of enforced dynamics. Analyzing the ambit of performances given on the mixed $\mathrm{H}_{2} / \mathrm{H}_{\infty}$ design, the joint design conditions are formulated as a minimization problem subject to convex constraints expressed by a system of LMIs. The feasibility of the conditions is demonstrated by a numerical example.
\end{abstract}

Key words: linear systems, fault tolerant control, fault estimation, linear matrix inequalities, $\mathrm{H}_{\infty}$ norm, $\mathrm{H}_{2} / \mathrm{H}_{\infty}$ control strategy.

\section{Introduction}

A model-based fault tolerant control (FTC) can be realized as control-laws set dependent, exploiting fault detection and isolation decision to reconfigure the control structure or as fault estimation dependent, preferring fault compensation within robust control framework. Whilst integration of FTC with the fault localization decision technique requires a selection of optimal residual thresholds as well as a robust and stable reconfiguration mechanism [5], the fault estimation dependent FTC structures eliminate a threshold subjectivism and integrate FTC and estimation problems into one robust optimization task [17], [18].

The $\mathrm{H}_{2}$-norm is one of the most important characteristics for linear time-invariant control systems, and is often used as performance index of the system in analysis and

The authors are with Technical University of Košice, Faculty of Electrical Engineering and Informatics, Department of Cybernetics and Artificial Intelligence, Letná 9, 04200 Košice, Slovakia, fax: +421 55625 3574, e-mail: dusan.krokavec@tuke.sk, anna.filasova@tuke.sk, pavol.liscinsky@tuke.sk

The work presented in this paper was supported by VEGA, the Grant Agency of the Ministry of Education and the Academy of Science of Slovak Republic, under Grant No. 1/0348/14. This support is very gratefully acknowledged.

Received 19.06.2016. Revised 6.11.2016. 
design problems. Thus, problems concerning $\mathrm{H}_{2}$, as well as $\mathrm{H}_{\infty}$ control have been studied by many authors [9], [16], [20], [21]. Adding $\mathrm{H}_{2}$ objective to $\mathrm{H}_{\infty}$ control design, a mixed $\mathrm{H}_{2} / \mathrm{H}_{\infty}$ control problem was formulated in [13], with the goal to design the gain matrix of the state-feedback control law such that minimizes $\mathrm{H}_{2}$ norm subject the constraint on $\mathrm{H}_{\infty}$ norm of the disturbance transfer function. Such integrated design strategy corresponds to optimization of the design parameters to satisfy desired specifications and to optimize the performance of the closed-loop system. Because of the importance of control systems with these properties, considerable attention was dedicated to mixed $\mathrm{H}_{2} / \mathrm{H}_{\infty}$ closed-loop performance criterion in design [1], [7], [10], [23], as well as to formulate the LMI-based computational technique [19], [24] to solve them or to exploit non-linear multi-objective algorithms for non-smooth optimization in this design task [11].

The approach, in which faults estimates are used in the control structure to compensate the effects of acting faults, is adopted in modern FTC techniques [3], [8], [28]. Integrated single-step methods of fault estimation and FTC, for linear systems subject to bounded actuator or sensor faults, are proposed in [17], [27]. Specified via LMI formulations and solved using $\mathrm{H}_{\infty}$ or mixed $\mathrm{H}_{2} / \mathrm{H}_{\infty}$ optimization, the observer structures are augmented fault state observers in the standard Luenberger form [22] or unknown input augmented fault observers [25]. To guarantee desired time response, an LMI regional pole placement design strategy is proposed in [26], [27]. However, such formulation introduces minimally two additive LMIs, which increase conservatism of the design conditions. Moreover, due to the extended structure of the parameter matrices of the fault tolerant controller [17], the LMI-based $\mathrm{H}_{\infty}$ control design solutions are, in general, marginal feasible.

Re-modifying the results given in [4] by an updated $\mathcal{D}$-stability circle criterion [14], the LMI conditions for fault observer design, proposed in this paper, inherently includes within minimal set of LMIs the regional pole placement condition to guarantee a suitable fault observer dynamics and, in consequence, a satisfactory time response of the FTC structure. Moreover, formulating as a problem subject to convex constraints, a mixed $\mathrm{H}_{2} / \mathrm{H}_{\infty}$ standard design method presented in [20] is modified relative the dual property of Schur complements. Accordingly, since an extended Lyapunov function is exploited, the obtained $\mathrm{H}_{2} / \mathrm{H}_{\infty}$ design conditions are regularized under acting of $\mathrm{H}_{2}$ constraint.

The content and scope of the paper are as follows. Placed after the introduction, presented in Sec. 1, the problem formulation and the basic preliminaries are given in Sec. 2 Next, Sec. 3 recalls the formulation of the fault-augmented observer for continuous-time linear systems and the controller design conditions in the framework of LMIs are recast in Sec. 4 Then, in Sec. 5, in response to fault compensation principle for such type of fault observers, the design conditions for the fault tolerant tracking control structures are derived, reflecting the mixed $\mathrm{H}_{2} / \mathrm{H}_{\infty}$ control idea. The relevance of the proposed approach is illustrated by a numerical example in Sec. 6 and Sec. 7 draws some concluding remarks.

Throughout the paper, the notations is narrowly standard in such a way that $\boldsymbol{x}^{T}, \boldsymbol{X}^{T}$ denotes the transpose of the vector $\boldsymbol{x}$ and matrix $\boldsymbol{X}$, respectively, $\operatorname{diag}(\cdot)$ denotes a block diagonal matrix, $\operatorname{rank}(\cdot)$ remits the rank of a matrix, for a square matrix $\boldsymbol{X}<0$ means 
that $\boldsymbol{X}$ is a symmetric negative definite matrix, the symbol $\boldsymbol{I}_{n}$ indicates the $n$-th order unit matrix, $\Re$ denotes the set of real numbers and $\Re^{n \times r}$ refers to the set of all $n \times r$ real matrices.

\section{Basic preliminaries}

The considered systems are described in the state-space form by the set of equations

$$
\begin{gathered}
\dot{\boldsymbol{q}}(t)=\boldsymbol{A} \boldsymbol{q}(t)+\boldsymbol{B} \boldsymbol{u}(t)+\boldsymbol{F} \boldsymbol{f}(t), \\
\boldsymbol{y}(t)=\boldsymbol{C} \boldsymbol{q}(t),
\end{gathered}
$$

where $\boldsymbol{q}(t) \in \mathfrak{R}^{n}, \boldsymbol{u}(t) \in \mathfrak{R}^{r}, \boldsymbol{y}(t) \in \mathfrak{R}^{m}$ are the vectors of the state, input and output variables, $\boldsymbol{f}(t) \in \mathfrak{R}^{p}$ is the fault vector, $\boldsymbol{A} \in \mathfrak{R}^{n \times n}, \boldsymbol{B} \in \mathfrak{R}^{n \times r}, \boldsymbol{C} \in \mathfrak{R}^{m \times n}, \boldsymbol{F} \in \mathfrak{R}^{n \times p}$ are real finite values matrices, $m, r, p<n$ and

$$
\operatorname{rank}\left[\begin{array}{cc}
\boldsymbol{A} & \boldsymbol{F} \\
\boldsymbol{C} & \mathbf{0}
\end{array}\right]=n+p .
$$

The transfer function matrix with respect to (1), (2) is

$$
\boldsymbol{G}(s)=\boldsymbol{C}\left(s \boldsymbol{I}_{n}-\boldsymbol{A}\right)^{-1} \boldsymbol{B},
$$

which gives the relationship to the state representation.

It is assumed that the fault $\boldsymbol{f}(t)$ may occur at an uncertain time, is slowly-varying and bounded and, to estimate such faults, it is supposed that the pair $(\boldsymbol{A}, \boldsymbol{C})$ is observable.

In order to analyze whether a system is stable under defined quadratic constraints, the concept can be summarized by the following LMI forms.

Lemma 1 [6] The matrix $\boldsymbol{A}$ is Hurwitz and $\|\boldsymbol{G}(s)\|_{2}<\gamma_{2}$ if there exists a symmetric positive definite matrix $\boldsymbol{V} \in \mathfrak{R}^{n \times n}$ and a positive scalar $\gamma_{2} \in \mathfrak{R}$, such that

$$
\begin{gathered}
\boldsymbol{V}=\boldsymbol{V}^{T}>0, \\
\boldsymbol{A} \boldsymbol{V}+\boldsymbol{V} \boldsymbol{A}^{T}+\boldsymbol{B} \boldsymbol{B}^{T}<0, \\
\operatorname{tr}\left(\boldsymbol{C} \boldsymbol{V} \boldsymbol{C}^{T}\right)<\gamma_{2}^{2},
\end{gathered}
$$

where $\gamma_{2}>0, \gamma_{2} \in \Re$ is $H_{2}$ norm of the transfer function matrix (4) of the system.

Lemma 2 [2] The matrix $\boldsymbol{A}$ is Hurwitz and $\|\boldsymbol{G}(s)\|_{\infty}<\gamma_{\infty}$ if there exists a symmetric positive definite matrix $\boldsymbol{R} \in \mathfrak{R}^{n \times n}$ and a positive scalar $\gamma_{\infty} \in \mathfrak{R}$ such that

$$
\boldsymbol{R}=\boldsymbol{R}^{T}>0
$$




$$
\left[\begin{array}{ccc}
\boldsymbol{A}^{T} \boldsymbol{R}+\boldsymbol{R A} & * & * \\
\boldsymbol{B}^{T} \boldsymbol{R} & -\gamma_{\infty} \boldsymbol{I}_{r} & * \\
\boldsymbol{C} & \mathbf{0} & -\gamma_{\infty} \boldsymbol{I}_{m}
\end{array}\right]<0,
$$

where $\boldsymbol{I}_{r} \in \mathfrak{R}^{r \times r}, \boldsymbol{I}_{m} \in \mathfrak{R}^{m \times m}$ are identity matrices and $\gamma_{\infty}>0, \gamma_{\infty} \in \mathfrak{R}$ is $H_{\infty}$ norm of the transfer function matrix (4) of the system.

Hereafter, $*$ denotes the symmetric item in a symmetric matrix.

Lemma 3 [14] The matrix $\boldsymbol{A}$ is $\mathcal{D}$-stable Hurwitz iffor given positive scalars a, $\rho \in \mathfrak{R}$, $a>\rho>0$, there exists a symmetric positive definite matrix $\boldsymbol{P} \in \mathfrak{R}^{n \times n}$ such that

$$
\left[\begin{array}{cc}
-\rho \boldsymbol{P} & * \\
\boldsymbol{P A}+a \boldsymbol{P} & -\rho \boldsymbol{P}
\end{array}\right]<0,
$$

while the eigenvalues of $\boldsymbol{A}$ are clustered in the circle with the origin $s_{o}=(-a+0 i)$ and radius $\rho$ within the complex plane $S$.

\section{Observer-based fault estimation}

Limiting to time invariant systems and focusing on fault estimation for slowlyvarying faults, the fault observer is considered in the following form

$$
\begin{aligned}
& \dot{\boldsymbol{q}}_{e}(t)=\boldsymbol{A} \boldsymbol{q}_{e}(t)+\boldsymbol{B u}(t)+\boldsymbol{F} \boldsymbol{f}_{e}(t)+\boldsymbol{J}\left(\boldsymbol{y}(t)-\boldsymbol{y}_{e}(t)\right), \\
& \boldsymbol{y}_{e}(t)=\boldsymbol{C} \boldsymbol{q}_{e}(t),
\end{aligned}
$$

where $\boldsymbol{q}_{e}(t) \in \mathfrak{R}^{n}, \boldsymbol{y}_{e}(t) \in \mathfrak{R}^{m}, \boldsymbol{f}_{e}(t) \in \mathfrak{R}^{p}$ are estimates of the system states vector, the output variables vector and the fault vector, respectively, and $\boldsymbol{J} \in \mathfrak{R}^{n \times m}$ is the observer gain matrix.

If the observer errors between the system state vector and the observer state vector, as well as between the fault vector and the vector of its estimate, are defined as follows

$$
\boldsymbol{e}_{q}(t)=\boldsymbol{q}(t)-\boldsymbol{q}_{e}(t), \quad \boldsymbol{e}_{f}(t)=\boldsymbol{f}(t)-\boldsymbol{f}_{e}(t),
$$

it is reasonable to consider for slowly-varying faults [27]

$$
\dot{\boldsymbol{f}}(t) \approx \mathbf{0}, \quad \dot{\boldsymbol{f}}_{e}(t)=\boldsymbol{L} \boldsymbol{C} \boldsymbol{e}_{q}(t),
$$

where $\boldsymbol{L} \in \Re^{p \times m}$ is the design parameter. The goal is to synthesize the couple $(\boldsymbol{J}, \boldsymbol{L})$ in such a way that the fault observer (11)-(14) is stable.

In order to be able to formulate the fault observer design conditions, (1), (2) and (14) are rewritten compositely as

$$
\left[\begin{array}{c}
\dot{\boldsymbol{q}}(t) \\
\dot{\boldsymbol{f}}(t)
\end{array}\right]=\left[\begin{array}{cc}
\boldsymbol{A} & \boldsymbol{F} \\
\mathbf{0} & \mathbf{0}
\end{array}\right]\left[\begin{array}{l}
\boldsymbol{q}(t) \\
\boldsymbol{f}(t)
\end{array}\right]+\left[\begin{array}{c}
\boldsymbol{B} \\
\mathbf{0}
\end{array}\right] \boldsymbol{u}(t),
$$




$$
\boldsymbol{y}(t)=\left[\begin{array}{ll}
\boldsymbol{C} & \mathbf{0}
\end{array}\right]\left[\begin{array}{l}
\boldsymbol{q}(t) \\
\boldsymbol{f}(t)
\end{array}\right]
$$

and, analogously, (11), (12), (14) as

$$
\begin{gathered}
{\left[\begin{array}{c}
\dot{\boldsymbol{q}}_{e}(t) \\
\dot{\boldsymbol{f}}_{e}(t)
\end{array}\right]=\left[\begin{array}{cc}
\boldsymbol{A} & \boldsymbol{F} \\
\mathbf{0} & \mathbf{0}
\end{array}\right]\left[\begin{array}{l}
\boldsymbol{q}_{e}(t) \\
\boldsymbol{f}_{e}(t)
\end{array}\right]+\left[\begin{array}{l}
\boldsymbol{B} \\
\boldsymbol{0}
\end{array}\right] \boldsymbol{u}(t)+\left[\begin{array}{l}
\boldsymbol{J} \\
\boldsymbol{L}
\end{array}\right] \boldsymbol{C} \boldsymbol{e}_{q}(t),} \\
\boldsymbol{y}_{e}(t)=\left[\begin{array}{ll}
\boldsymbol{C} & 0
\end{array}\right]\left[\begin{array}{l}
\boldsymbol{q}_{e}(t) \\
\boldsymbol{f}_{e}(t)
\end{array}\right] .
\end{gathered}
$$

Thus, introducing the notations

$$
\begin{gathered}
\boldsymbol{q}^{\circ T}(t)=\left[\begin{array}{ll}
\boldsymbol{q}^{T}(t) & \boldsymbol{f}^{T}(t)
\end{array}\right], \quad \boldsymbol{q}_{e}^{\circ T}(t)=\left[\begin{array}{ll}
\boldsymbol{q}_{e}^{T}(t) & \boldsymbol{f}_{e}^{T}(t)
\end{array}\right], \\
\boldsymbol{A}^{\circ}=\left[\begin{array}{ll}
\boldsymbol{A} & \boldsymbol{F} \\
\mathbf{0} & \mathbf{0}
\end{array}\right], \boldsymbol{B}^{\circ}=\left[\begin{array}{l}
\boldsymbol{B} \\
\mathbf{0}
\end{array}\right], \boldsymbol{J}^{\circ}=\left[\begin{array}{l}
\boldsymbol{J} \\
\boldsymbol{L}
\end{array}\right], \boldsymbol{C}^{\circ}=\left[\begin{array}{ll}
\boldsymbol{C} & \mathbf{0}
\end{array}\right],
\end{gathered}
$$

where $\boldsymbol{A}^{\circ} \in \mathfrak{R}^{(n+p) \times(n+p)}, \boldsymbol{J}^{\circ} \in \mathfrak{R}^{(n+p) \times m}, \boldsymbol{C}^{\circ} \in \mathfrak{R}^{m \times(n+p)}, \boldsymbol{B}^{\circ} \in \mathfrak{R}^{(n+p) \times r}, \boldsymbol{q}^{\circ}(t), \boldsymbol{q}_{e}^{\circ}(t) \in$ $\mathfrak{R}^{n+p}$, then from the above follows

$$
\begin{gathered}
\dot{\boldsymbol{q}}^{\circ}(t)=\boldsymbol{A}^{\circ} \boldsymbol{q}^{\circ}(t)+\boldsymbol{B}^{\circ} \boldsymbol{u}(t), \\
\dot{\boldsymbol{q}}_{e}^{\circ}(t)=\boldsymbol{A}^{\circ} \boldsymbol{q}_{e}^{\circ}(t)+\boldsymbol{B}^{\circ} \boldsymbol{u}(t)+\boldsymbol{J}^{\circ}\left(\boldsymbol{y}(t)-\boldsymbol{y}_{e}(t)\right), \\
\boldsymbol{y}(t)=\boldsymbol{C}^{\circ} \boldsymbol{q}^{\circ}(t), \quad \boldsymbol{y}_{e}(t)=\boldsymbol{C}^{\circ} \boldsymbol{q}_{e}^{\circ}(t) .
\end{gathered}
$$

This leads to the following equation

$$
\dot{\boldsymbol{e}}^{\circ}(t)=\left(\boldsymbol{A}^{\circ}-\boldsymbol{J}^{\circ} \boldsymbol{C}^{\circ}\right) \boldsymbol{e}^{\circ}(t)=\boldsymbol{A}_{e}^{\circ} \boldsymbol{e}^{\circ}(t),
$$

where

$$
\begin{gathered}
\boldsymbol{A}_{e}^{\circ}=\boldsymbol{A}^{\circ}-\boldsymbol{J}^{\circ} \boldsymbol{C}^{\circ}, \\
\boldsymbol{e}^{\circ}(t)=\boldsymbol{q}^{\circ}(t)-\boldsymbol{q}_{e}^{\circ}(t),
\end{gathered}
$$

$\boldsymbol{q}_{e}^{\circ}(t)$ and $\boldsymbol{y}_{e}(t)$ represent the estimates of $\boldsymbol{q}^{\circ}(t)$ and $\boldsymbol{y}(t)$, respectively, and $\boldsymbol{e}^{\circ}(t)$ is the fault observer error. 


\section{Control design strategies}

It is assumed that the system (22), (23) is controllable to be controlled by the state feedback control law

$$
\begin{gathered}
\boldsymbol{u}(t)=-\boldsymbol{K}^{\circ} \boldsymbol{q}^{\circ}(t), \\
\boldsymbol{K}^{\circ}=\left[\begin{array}{ll}
\boldsymbol{K}_{q} & \boldsymbol{K}_{f}
\end{array}\right],
\end{gathered}
$$

where $\boldsymbol{K}^{\circ} \in \mathfrak{R}^{r \times(n+p)}$ is the control law gain matrix.

If the system set-point may vary under normal circumstances, it is desired to adjust the plant working point indirectly by the output following. That this be done, the difference between the output $\boldsymbol{y}(t)$ and its desired value $\boldsymbol{w}(t)$ has to be exploited so that the outputs could try to follow their desired values. It is possible to ensure this idea by the integral tracking, where the controller integral term minimized this difference. In order to eliminate tracking error, the control law (27) is extended by an integral component of the form [29]

$$
\boldsymbol{e}_{w}(t)=\int_{0}^{t}(\boldsymbol{w}(\tau)-\boldsymbol{y}(\tau)) \mathrm{d} \tau,
$$

which is joined to (27) in such a way that

$$
\boldsymbol{u}(t)=-\boldsymbol{K}^{\bullet} \boldsymbol{q}^{\bullet}(t),
$$

where

$$
\begin{aligned}
& \boldsymbol{q}^{\bullet T}(t)=\left[\begin{array}{ll}
\boldsymbol{q}^{\circ T}(t) & \boldsymbol{e}_{w}^{T}(t)
\end{array}\right]=\left[\begin{array}{lll}
\boldsymbol{q}^{T}(t) & \boldsymbol{f}^{T}(t) & \boldsymbol{e}_{w}^{T}(t)
\end{array}\right], \\
& \boldsymbol{K}^{\bullet}=\left[\begin{array}{ll}
\boldsymbol{K}^{\circ} & \boldsymbol{K}_{w}
\end{array}\right]=\left[\begin{array}{lll}
\boldsymbol{K}_{q} & \boldsymbol{K}_{f} & \boldsymbol{K}_{w}
\end{array}\right],
\end{aligned}
$$

while $\boldsymbol{q}^{\bullet}(t) \in \mathfrak{R}^{n+p+m}, \boldsymbol{K}^{\bullet} \in \mathfrak{R}^{r \times(n+p+m)}$.

From (29) follows directly

$$
\dot{\boldsymbol{e}}_{w}(t)=\boldsymbol{w}(t)-\boldsymbol{y}(t)
$$

and the system model (15), (16) has to be expanded as

$$
\begin{gathered}
{\left[\begin{array}{c}
\dot{\boldsymbol{q}}(t) \\
\dot{\boldsymbol{f}}(t) \\
\dot{\boldsymbol{e}}_{w}(t)
\end{array}\right]=\left[\begin{array}{rrr}
\boldsymbol{A} & \boldsymbol{F} & \mathbf{0} \\
\mathbf{0} & \mathbf{0} & \mathbf{0} \\
-\boldsymbol{C} & \mathbf{0} & \mathbf{0}
\end{array}\right]\left[\begin{array}{c}
\boldsymbol{q}(t) \\
\boldsymbol{f}(t) \\
\boldsymbol{e}_{w}(t)
\end{array}\right]+\left[\begin{array}{c}
\boldsymbol{B} \\
\mathbf{0} \\
\mathbf{0}
\end{array}\right] \boldsymbol{u}(t)+\left[\begin{array}{c}
\mathbf{0} \\
\mathbf{0} \\
\boldsymbol{I}_{m}
\end{array}\right] \boldsymbol{w}(t),} \\
\boldsymbol{y}(t)=\left[\begin{array}{lll}
\boldsymbol{C} & \mathbf{0} & \mathbf{0}
\end{array}\right]\left[\begin{array}{c}
\boldsymbol{q}(t) \\
\boldsymbol{f}(t) \\
\boldsymbol{e}_{w}(t)
\end{array}\right]
\end{gathered}
$$


where $\boldsymbol{I}_{m}$ is the identity matrix of given dimension.

Using the notations (31), (32) and

$$
A^{\bullet}=\left[\begin{array}{rrr}
A & F & 0 \\
0 & 0 & 0 \\
-C & 0 & 0
\end{array}\right], B^{\bullet}=\left[\begin{array}{l}
B \\
0 \\
0
\end{array}\right], W^{\bullet}=\left[\begin{array}{c}
0 \\
0 \\
I_{m}
\end{array}\right], C^{\bullet}=\left[\begin{array}{lll}
C & 0 & 0
\end{array}\right],
$$

then the state-space description of the closed-loop system (34), (35), (30) can be written as

$$
\begin{gathered}
\dot{\boldsymbol{q}}^{\bullet}(t)=\boldsymbol{A}_{c}^{\bullet} \boldsymbol{q}^{\bullet}(t)+\boldsymbol{W}^{\bullet} \boldsymbol{w}(t), \\
\boldsymbol{y}(t)=\boldsymbol{C}^{\bullet} \boldsymbol{q}^{\bullet}(t),
\end{gathered}
$$

where

$$
\boldsymbol{A}_{c}^{\bullet}=A^{\bullet}-B^{\bullet} K^{\bullet}
$$

is the closed-loop system matrix of the expanded system.

In order to test whether the system is controllable with reference attenuations $\gamma_{\infty}$, the transfer function matrices

$$
\begin{aligned}
\boldsymbol{G}^{\bullet}(s) & =\boldsymbol{C}^{\bullet}\left(s \boldsymbol{I}_{n+p+m}-\boldsymbol{A}_{c}^{\bullet}\right)^{-1} \boldsymbol{B}^{\bullet}, \\
\boldsymbol{G}_{w}(s) & =\boldsymbol{C}^{\bullet}\left(s \boldsymbol{I}_{n+p+m}-\boldsymbol{A}_{c}^{\bullet}\right)^{-1} \boldsymbol{W}^{\bullet},
\end{aligned}
$$

are considered in the following.

Lemma 4 ( $\mathrm{H}_{2}$ control synthesis) The state feedback control (30) to the system (37), (38) exists and $\left\|\boldsymbol{G}^{\bullet}(s)\right\|_{2}<\gamma_{2}^{\bullet}$ if there exist symmetric positive definite matrices $\boldsymbol{V}^{\bullet} \in$ $\mathfrak{R}^{(n+p+m) \times(n+p+m)}, \boldsymbol{H}^{\bullet} \in \mathfrak{R}^{m \times m}$, a matrix $\boldsymbol{Y}^{\bullet} \in \mathfrak{R}^{r \times(n+p+m)}$ and a positive scalar $\eta^{\bullet} \in \mathfrak{R}$ such that

$$
\begin{gathered}
\boldsymbol{V}^{\bullet}=\boldsymbol{V}^{\bullet T}>0, \quad \boldsymbol{H}^{\bullet}=\boldsymbol{H}^{\bullet T}>0, \\
{\left[\begin{array}{rr}
\boldsymbol{A}^{\bullet} \boldsymbol{V}^{\bullet}+\boldsymbol{V}^{\bullet} \boldsymbol{A}^{\bullet T}-\boldsymbol{B}^{\bullet} \boldsymbol{Y}^{\bullet}-\boldsymbol{Y}^{\bullet T} \boldsymbol{B}^{\bullet T} & * \\
\boldsymbol{B}^{\bullet T} & -\boldsymbol{I}_{r}
\end{array}\right]<0,} \\
{\left[\begin{array}{cc}
\boldsymbol{V}^{\bullet} & * \\
\boldsymbol{C}^{\bullet} \boldsymbol{V}^{\bullet} & \boldsymbol{H}^{\bullet}
\end{array}\right]>0,} \\
\operatorname{tr}\left(\boldsymbol{H}^{\bullet}\right)<\eta^{\bullet}
\end{gathered}
$$

When the above conditions hold, the control law gain is

$$
\boldsymbol{K}^{\bullet}=\boldsymbol{Y}^{\bullet}\left(\boldsymbol{V}^{\bullet}\right)^{-1}
$$

Proof Rearranging the inequality (6) by using Schur complement property, it yields

$$
\left[\begin{array}{cc}
\boldsymbol{A} \boldsymbol{V}+\boldsymbol{V} \boldsymbol{A}^{T} & \boldsymbol{B} \\
\boldsymbol{B}^{T} & -\boldsymbol{I}_{r}
\end{array}\right]<0 .
$$


Replacing $\boldsymbol{A}$ in (46) by (40) and $\boldsymbol{V}$ by $\boldsymbol{V}^{\bullet}$ redefines the linear matrix inequality (46) as follows

$$
\left[\begin{array}{cc}
\left(\boldsymbol{A}^{\bullet}-\boldsymbol{B}^{\bullet} \boldsymbol{K}^{\bullet}\right) \boldsymbol{V}^{\bullet}+\boldsymbol{V}^{\bullet}\left(\boldsymbol{A}^{\bullet}-\boldsymbol{B}^{\bullet} \boldsymbol{K}^{\bullet}\right)^{T} & \boldsymbol{B}^{\bullet} \\
\boldsymbol{B}^{\bullet T} & -\boldsymbol{I}_{r}
\end{array}\right]<0
$$

so, with the notation

$$
\boldsymbol{Y}^{\bullet}=\boldsymbol{K}^{\bullet} \boldsymbol{V}^{\bullet}
$$

(47) implies (43).

The objective of $\mathrm{H}_{2}$ control is to minimize the constraint

$$
\operatorname{tr}\left(\boldsymbol{C}^{\bullet} \boldsymbol{V}^{\bullet} \boldsymbol{C}^{\bullet T}\right)<\left(\gamma_{2}^{\bullet}\right)^{2}
$$

but this inequality cannot be directly optimized. Introducing the inequality

$$
\boldsymbol{H}^{\bullet}>\boldsymbol{C}^{\bullet} \boldsymbol{V}^{\bullet} \boldsymbol{C}^{\bullet T}=\boldsymbol{C}^{\bullet} \boldsymbol{V}^{\bullet}\left(\boldsymbol{V}^{\bullet}\right)^{-1} \boldsymbol{V}^{\bullet} \boldsymbol{C}^{\bullet T}, \quad \operatorname{tr}\left(\boldsymbol{H}^{\bullet}\right)=\eta^{\bullet},
$$

with a new matrix variable $\boldsymbol{H}^{\bullet}$ being symmetric and positive definite, and using Schur complement property, then (49), (50) imply directly (44). This concludes the proof.

Note, to obtain a feasible block structure of the LMI, Schur complement property has to be used. Contrarily to the algorithm presented in [20], the Schur complement property was used to rearrange (6) to obtain (46) while the dual Schur complement property was applied to modify (49). It is the main reason that the proof is attached to this lemma.

Lemma 5 ( $H_{\infty}$ control synthesis) The state feedback control (30) to the system (37), (38) exists and $\left\|\boldsymbol{G}_{w}^{\bullet}(s)\right\|_{\infty}<\gamma_{\infty}$ if there exist a symmetric positive definite matrix $\boldsymbol{Q}^{\bullet} \in$ $\mathfrak{R}^{(n+p+m) \times(n+p+m)}$, a matrix $\mathbf{Z}^{\bullet} \in \mathfrak{R}^{r \times(n+p+m)}$ and a positive scalar $\gamma_{\infty}^{\bullet} \in \mathfrak{R}$ such that

$$
\begin{gathered}
\boldsymbol{Q}^{\bullet}=\boldsymbol{Q}^{\bullet T}>0, \\
{\left[\begin{array}{ccc}
\boldsymbol{A}^{\bullet} \boldsymbol{Q}^{\bullet}+\boldsymbol{Q}^{\bullet} \boldsymbol{A}^{\bullet T}-\boldsymbol{B}^{\bullet} \boldsymbol{Z}^{\bullet}-\boldsymbol{Z}^{\bullet T} \boldsymbol{B}^{\bullet T} & * & * \\
\boldsymbol{W}^{\bullet T} & -\gamma_{\infty} \boldsymbol{I}_{m} & * \\
\boldsymbol{C}^{\bullet} \boldsymbol{Q}^{\bullet} & \mathbf{0} & -\gamma_{\infty} \boldsymbol{I}_{m}
\end{array}\right]<0 .}
\end{gathered}
$$

When the above conditions hold, the control law gain is

$$
\boldsymbol{K}^{\bullet}=\mathbf{Z}^{\bullet}\left(\boldsymbol{Q}^{\bullet}\right)^{-1}
$$

Proof Replacing in (9) $\boldsymbol{A}$ by $\boldsymbol{A}_{c}^{\bullet}, \boldsymbol{R}$ by $\boldsymbol{R}^{\bullet}, \boldsymbol{B}$ by $\boldsymbol{W}^{\bullet}$ and $\boldsymbol{I}_{r}$ by $\boldsymbol{I}_{m}$, and defining the transform matrix

$$
\boldsymbol{T}^{\bullet}=\operatorname{diag}\left[\begin{array}{lll}
\boldsymbol{Q}^{\bullet} & \boldsymbol{I}_{m} & \boldsymbol{I}_{m}
\end{array}\right], \quad \boldsymbol{Q}^{\bullet}=\left(\boldsymbol{R}^{\bullet}\right)^{-1},
$$


then pre-multiplying the left side and post-multiplying the ride side of (9) by $\boldsymbol{T}^{\bullet}$, it yields

$$
\left[\begin{array}{ccc}
\boldsymbol{A}_{c}^{\bullet} \boldsymbol{Q}^{\bullet}+\boldsymbol{Q}^{\bullet} \boldsymbol{A}_{c}^{\bullet T} & * & * \\
\boldsymbol{W}^{\bullet T} & -\gamma_{\infty} \boldsymbol{I}_{m} & * \\
\boldsymbol{C}^{\bullet} \boldsymbol{Q}^{\bullet} & \mathbf{0} & -\gamma_{\infty} \boldsymbol{I}_{m}
\end{array}\right]<0
$$

Substituting (39) modifies the linear matrix inequality (55) as follows

$$
\left[\begin{array}{ccc}
\left(\boldsymbol{A}^{\bullet}-\boldsymbol{B}^{\bullet} \boldsymbol{K}^{\bullet}\right) \boldsymbol{Q}^{\bullet}+\boldsymbol{Q}^{\bullet}\left(\boldsymbol{A}^{\bullet}-\boldsymbol{B}^{\bullet} \boldsymbol{K}^{\bullet}\right)^{T} & * & * \\
\boldsymbol{W}^{\bullet T} & -\gamma_{\infty} \boldsymbol{I}_{m} & * \\
\boldsymbol{C}^{\bullet} \boldsymbol{Q}^{\bullet} & 0 & -\gamma_{\infty} \boldsymbol{I}_{m}
\end{array}\right]<0
$$

and with the notation

$$
Z^{\bullet}=K^{\bullet} Q^{\bullet}
$$

(56) implies (52). This concludes the proof.

It is important to point out, due to the structure of the matrix $\boldsymbol{A}^{\bullet}, \boldsymbol{B}^{\bullet}$ that a solution of (51), (52) is usually marginal feasible [15], i.e., $\operatorname{rank}\left(\boldsymbol{A}^{\bullet}-\boldsymbol{B}^{\bullet} \boldsymbol{K}^{\bullet}\right)<n+p+m$, and the design has to be combined with any constraint. For this reason the proof for this lemma is given. The mixed $\mathrm{H}_{2} / \mathrm{H}_{\infty}$ control principle, using $\mathrm{H}_{2}$ and $\mathrm{H}_{\infty}$ performance constraints to solve FTC problem, is proposed in [27].

\section{Joint design of fault tolerant control}

With these expressions it is now easy to formulate the joint approach for integrated design of fault estimation and FTC, where $\boldsymbol{q}^{\bullet T}(t)$ is considered as

$$
\boldsymbol{q}^{\bullet T}(t)=\left[\begin{array}{lll}
\boldsymbol{q}^{T}(t) & \boldsymbol{f}_{e}^{T}(t) & \boldsymbol{e}_{w}^{T}(t)
\end{array}\right]
$$

Theorem 1 The state feedback control (30), (58) to the system (38), (39) exists and $\left\|\boldsymbol{G}^{\bullet}(s)\right\|_{2}<\gamma_{2}$ as well as $\left\|\boldsymbol{G}_{w}^{\bullet}(s)\right\|_{\infty}<\gamma_{\infty}$ if for given positive scalars $a, \rho \in \mathfrak{R}, a>$ $\rho>0$, there exist symmetric positive definite matrices $\boldsymbol{V}^{\bullet} \in \mathfrak{R}^{(n+p+m) \times(n+p+m)}, \boldsymbol{P}^{\bullet} \in$ $\mathfrak{R}^{(n+p) \times(n+p)}, \boldsymbol{H}^{\bullet} \in \mathfrak{R}^{m \times m}$, matrices $\boldsymbol{Y}^{\bullet} \in \mathfrak{R}^{r \times(n+p+m)}, \boldsymbol{S}^{\circ} \in \mathfrak{R}^{(n+p) \times m}$ and a positive scalar $\gamma_{\infty}^{\bullet} \in \mathfrak{R}$ such that

$$
\begin{gathered}
\boldsymbol{V}^{\bullet}=\boldsymbol{V}^{\bullet T}>0, \quad \boldsymbol{P}^{\bullet}=\boldsymbol{P}^{\bullet T}>0, \quad \boldsymbol{H}^{\bullet}=\boldsymbol{H}^{\bullet T}>0, \\
{\left[\begin{array}{cc}
-\rho \boldsymbol{P}^{\circ} & * \\
\boldsymbol{P}^{\circ} \boldsymbol{A}^{\circ}-\boldsymbol{S}^{\circ} \boldsymbol{C}^{\circ}+a \boldsymbol{P}^{\circ} & -\rho \boldsymbol{P}^{\circ}
\end{array}\right]<0,}
\end{gathered}
$$




$$
\begin{aligned}
& {\left[\begin{array}{ccc}
\boldsymbol{A}^{\bullet} \boldsymbol{V}^{\bullet}+\boldsymbol{V}^{\bullet} \boldsymbol{A}^{\bullet T}-\boldsymbol{B}^{\bullet} \boldsymbol{Y}^{\bullet}-\boldsymbol{Y}^{\bullet T} \boldsymbol{B}^{\bullet T} & * & * \\
\boldsymbol{W}^{\bullet T} & -\gamma_{\infty} \boldsymbol{I}_{m} & * \\
\boldsymbol{C}^{\bullet} \boldsymbol{V}^{\bullet} & \mathbf{0} & -\gamma_{\infty} \boldsymbol{I}_{m}
\end{array}\right]<0,} \\
& {\left[\begin{array}{cc}
\boldsymbol{A}^{\bullet} \boldsymbol{V}^{\bullet}+\boldsymbol{V}^{\bullet} \boldsymbol{A}^{\bullet T}-\boldsymbol{B}^{\bullet} \boldsymbol{Y}^{\bullet}-\boldsymbol{Y}^{\bullet T} \boldsymbol{B}^{\bullet T} & * \\
\boldsymbol{B}^{\bullet T} & -\boldsymbol{I}_{r}
\end{array}\right]<0,} \\
& {\left[\begin{array}{cc}
\boldsymbol{V}^{\bullet} & * \\
\boldsymbol{C}^{\bullet} \boldsymbol{V}^{\bullet} & \boldsymbol{H}^{\bullet}
\end{array}\right]>0, \quad \operatorname{tr}\left(\boldsymbol{H}^{\bullet}\right)<\eta^{\bullet} .}
\end{aligned}
$$

When the above conditions hold

$$
\boldsymbol{K}^{\bullet}=\boldsymbol{Y}^{\bullet}\left(\boldsymbol{V}^{\bullet}\right)^{-1}, \quad \boldsymbol{J}^{\circ}=\left(\boldsymbol{P}^{\circ}\right)^{-1} \boldsymbol{S}^{\circ} .
$$

Proof Replacing in (10) $\boldsymbol{A}$ by $\boldsymbol{A}_{e}^{\circ}$ and $\boldsymbol{P}$ by $\boldsymbol{P}^{\circ}$ results

$$
\left[\begin{array}{cc}
-\rho \boldsymbol{P}^{\circ} & * \\
\boldsymbol{P}^{\circ}\left(\boldsymbol{A}^{\circ}-\boldsymbol{J}^{\circ} \boldsymbol{C}^{\circ}\right)+a \boldsymbol{P}^{\circ} & -\rho \boldsymbol{P}^{\circ}
\end{array}\right]<0
$$

and with the notation

$$
\boldsymbol{S}^{\circ}=\boldsymbol{P}^{\circ} \boldsymbol{J}^{\circ}
$$

(65) implies (60).

Prescribing a unique solution of $\boldsymbol{K}^{\bullet}$ with respect to (45) and (53) that is

$$
\boldsymbol{V}^{\bullet}=\boldsymbol{Q}^{\bullet}, \quad \boldsymbol{Y}^{\bullet}=\mathbf{Z}^{\bullet},
$$

then (42)-(44) and (51), (52) in the joint sense implies (61)-(64). This concludes the proof.

Note, the introduced mixed $\mathrm{H}_{2} / \mathrm{H}_{\infty}$ control maximizes the $\mathrm{H}_{2}$ norm over all statefeedback gains $\boldsymbol{K}^{\bullet}$ while the $\mathrm{H}_{\infty}$ norm constraint is minimized. Comparing with [20], the set of LMIs (61)-(63) is well conditioned and feasible. The main reason for the use of $\mathrm{D}$-stability principle in the fault observer design is to adapt interactive the fault observer dynamics to the dynamics of the FTC structure.

\section{Illustrative example}

To illustrate the proposed method, a system whose dynamics is described by equations (1), (2) is considered with the matrix parameters [12]

$$
\boldsymbol{A}=\left[\begin{array}{rrrr}
1.380 & -0.208 & 6.715 & -5.676 \\
-0.581 & -4.290 & 0.000 & 0.675 \\
1.067 & 4.273 & -6.654 & 5.893 \\
0.048 & 4.273 & 1.343 & -2.104
\end{array}\right]
$$




$$
\boldsymbol{B}=\left[\begin{array}{rr}
0.000 & 0.000 \\
5.679 & 0.000 \\
1.136 & -3.146 \\
1.136 & 0.000
\end{array}\right], \boldsymbol{F}=\left[\begin{array}{l}
1.400 \\
1.504 \\
2.233 \\
0.610
\end{array}\right], \boldsymbol{C}=\left[\begin{array}{llll}
4 & 0 & 1 & 0 \\
0 & 0 & 0 & 1
\end{array}\right]
$$

Solving (59)-(63) using SeDuMi package, the design problem is solved as feasible where, with the prescribed stability region parameters $a=10, \rho=8.5$, the resulted control system parameters are

$$
\begin{aligned}
& \boldsymbol{K}_{q}=\left[\begin{array}{rrrr}
0.0709 & -0.7022 & 0.0245 & 1.9807 \\
-14.6220 & -0.0706 & -1.2190 & -1.2406
\end{array}\right], \\
& \boldsymbol{K}_{f}=\left[\begin{array}{r}
0.2546 \\
-0.5672
\end{array}\right], \boldsymbol{K}_{w}=\left[\begin{array}{rr}
-0.0859 & -0.3923 \\
0.9172 & 0.0261
\end{array}\right], \\
& \boldsymbol{J}=\left[\begin{array}{rr}
3.3817 & -2.7407 \\
0.4280 & 2.6900 \\
1.2510 & 7.4649 \\
0.5128 & 8.2180
\end{array}\right], \boldsymbol{L}=\left[\begin{array}{ll}
1.8415 & 3.1383
\end{array}\right], \boldsymbol{H}=\left[\begin{array}{rr}
31.3538 & 1.1875 \\
1.1875 & 21.0385
\end{array}\right] \text {, } \\
& \gamma_{\infty} \geqslant 21.1631, \quad \gamma_{2}^{2} \geqslant 28.0805,
\end{aligned}
$$

while

$$
\begin{gathered}
\rho\left(\boldsymbol{A}_{c}^{\bullet}\right)=\{0,-0.1784,-0.3017-1.6245 \pm 7.0670 \mathrm{i},-5.0321 \pm 16.4998 \mathrm{i}\}, \\
\rho\left(\boldsymbol{A}_{e}^{\circ}\right)=\{-3.4866-5.9462 \pm 1.1756 \mathrm{i},-9.6424 \pm 0.6226 \mathrm{i}\},
\end{gathered}
$$

which affirms that for given $(a, \rho)$ the dynamics of the fault augmented observer is substantially faster then the dynamics of the FTC structure.

The faults in simulations are generated using the window

$$
g(t)=\left\{\begin{array}{cl}
0, & t \leqslant t_{s a} \\
\frac{1}{t_{s b}-t_{s a}}\left(t-t_{s a}\right), & t_{s a}<t_{s b} \\
1, & t_{s b} \leqslant t_{e a} \\
-\frac{1}{t_{e b}-t_{e a}}\left(t-t_{e b}\right), & t_{e a}<t_{e b} \\
0, & t \geqslant t_{e b}
\end{array}\right.
$$

where it is adjusted

$$
\begin{gathered}
f_{1}(t)=g(t) \sin (\omega t), \quad \omega=0.2 \mathrm{rad} / \mathrm{sec}, \quad f_{2}(t)=g(t), \\
t_{s a}=30 \mathrm{~s}, \quad t_{s b}=35 \mathrm{~s}, \quad t_{e a}=65 \mathrm{~s}, \quad t_{e b}=70 \mathrm{~s},
\end{gathered}
$$


and the initial conditions are

$$
\boldsymbol{q}^{\bullet}(0)=\mathbf{0}, \boldsymbol{q}^{\circ}(0)=\mathbf{0}, \boldsymbol{w}^{T}(t)=\left[\begin{array}{ll}
1 & 2
\end{array}\right] .
$$

Figures 2-4 show the sinusoidal-like fault and its estimate, the corresponding estimated closed-loop system outputs as well as the corresponding closed-loop system outputs with fault compensation, when the sinusoidal-like fault arises. In the same manner, Figures 1-6 characterize the signals in the FTC structure with integrated fault estimation when the ramp fault comes into.

From the above given figures it is obvious that the integrated FTC, which parameters are obtained as a solution of the LMI problem specified by Theorem 1, can with sufficient precision approximate given class of slowly warring faults that their impact on the system output variables is successfully compensated. Moreover, the mixed joint design, which includes the updated $\mathcal{D}$-stability circle criterion, outperforms the two-stage design approach without increasing conservatism.

\section{Concluding remarks}

A modified approach for designing fault augmented observes, integrated with the compensation FTC structure, is presented in the contribution. Using LMI technique, the exploited mixed $\mathrm{H}_{2} / \mathrm{H}_{\infty}$ control design to regularize in general marginally feasible conditions, a dual form of the Schur complement to linearize the bilinear $\gamma_{2}$ constraint in LMI approach for $\mathrm{H}_{2}$ control principle, as well as an updated $\mathcal{D}$-stability circle criterion in fault observer design to adapt the fault observer dynamics to the dynamics of the control system, the design conditions are established as feasible problem, accomplishing under given quadratic constraints. Presented illustrative example confirms the effectiveness of the proposed design alternative, to construct the control structure with sufficient approximation of given class slowly warring faults and compensation of their impact on the system output variables. 


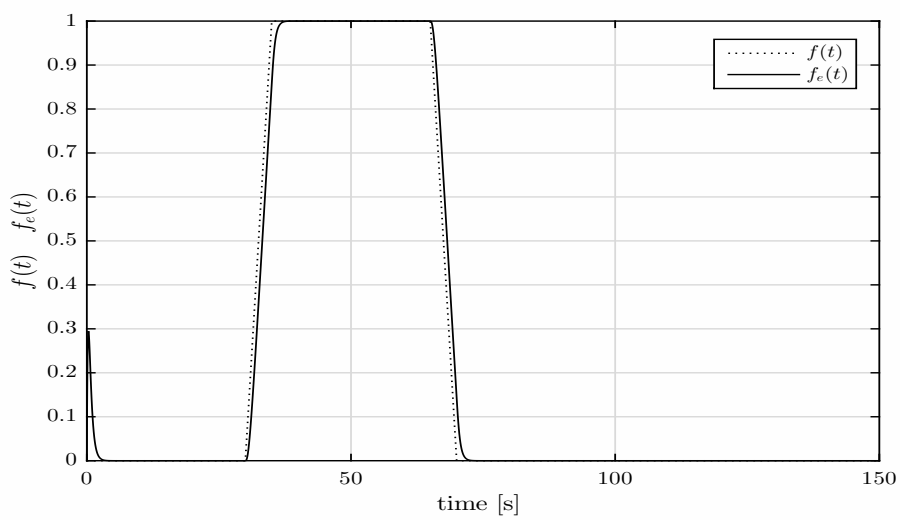

Figure 1: Estimated ramp fault

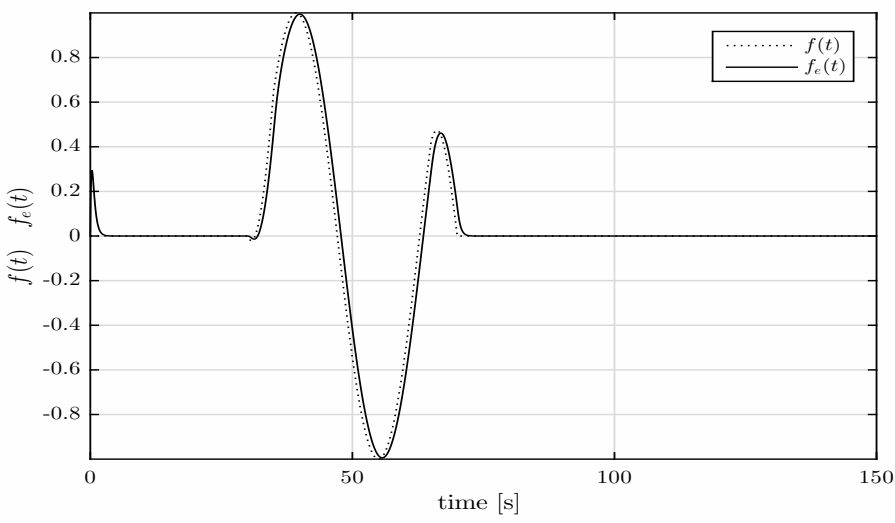

Figure 2: Estimated sinusoidal-like fault

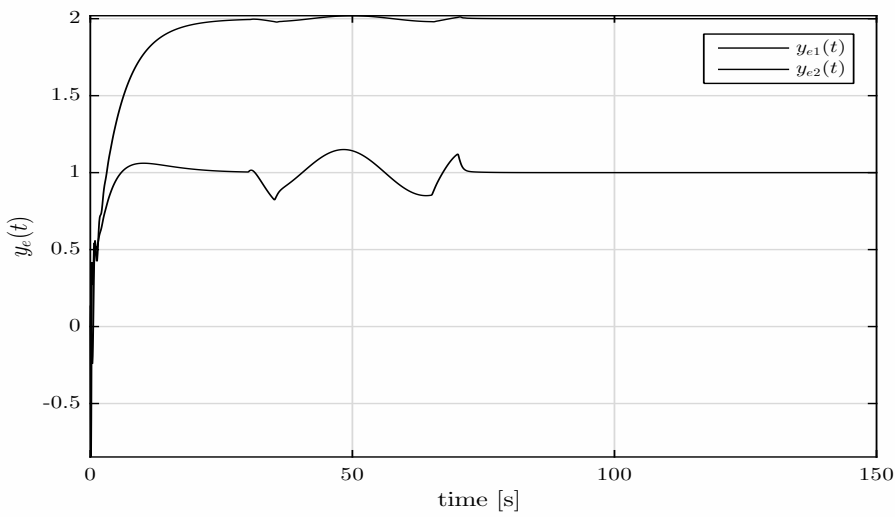

Figure 3: Estimated closed-loop system outputs 


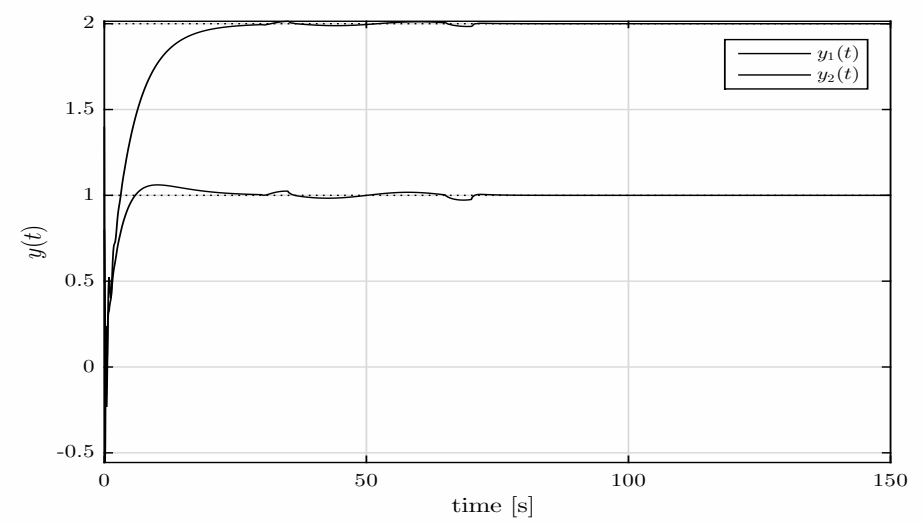

Figure 4: Closed-loop system outputs with fault compensation

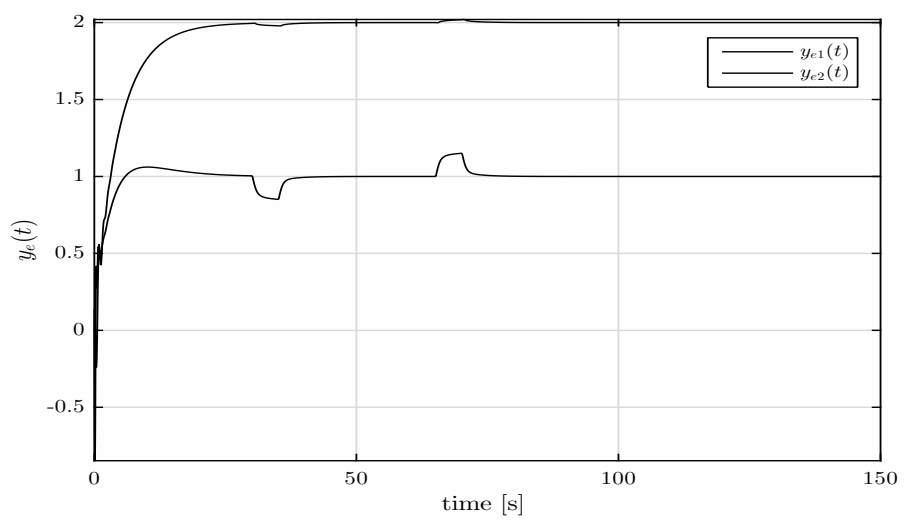

Figure 5: Estimated closed-loop system outputs

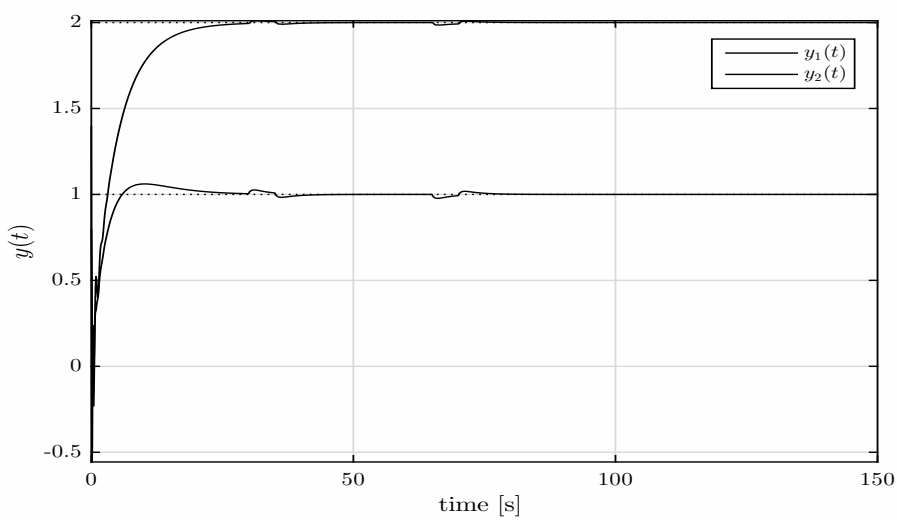

Figure 6: Closed-loop system outputs with fault compensation 


\section{References}

[1] R. Bambang, E. Shimemura and K. Uchjida: Mixed $\mathrm{H}_{2} / \mathrm{H}_{\infty}$ control with pole placement. State feedback case. Proc. 1993 American Control Conference, San Francisco, CA, USA, (1993), 2777-2779.

[2] D. BOYD and V. BALAKRISHNAN: A regularity result for the singular values of a transfer matrix and a qudratically convergent algorithm for computing its $\mathrm{L}_{\infty}$-norm. Systems \& Control Letters, 15(1), (1990), 1-7.

[3] Z. Cen, H. Noura and Y. Al Younes: Systematic fault tolerant control based on adaptive Thau observer estimation for quadrotor UAVs. Int. J. Applied Mathematics and Computer Science, 25(1), (2015), 159-174.

[4] M. Chilali and P. Gahinet: $\mathrm{H}_{\infty}$ design with pole placement constraints. An LMI Approch. Proc. 33rd Conf. Decision and Control, Lake Buena Vista, FL, USA, (1994), 553-558.

[5] S. Ding: Model-Based Fault Diagnosis Techniques. Design Schemes, Algorithms, and Tools, Berlin, Springer-Verlag, 2013.

[6] J. Doyle, B. Francis and A. Tannenbaum: Feedback Control Theory, New York, Macmillan Publishing, 1990.

[7] J.C. Doyle, K. Glover, P.P. Khargonekar and B.A. Francis: State-space solutions to standard $\mathrm{H}_{2}$ and $\mathrm{H}_{\infty}$ control problems. IEEE Tran. Automatic Control, 34(8), (1989), 831-847.

[8] A. Filasová and D. KroKaVeC: Design principles of active robust fault tolerant control systems. Robust Control. Theory and Applications, A. Bartoszewicz (Ed.), Rijeca, InTech, (2011), 309-338.

[9] B.A. Francis: A Course in $H_{\infty}$ Control Theory, Berlin, Springer-Verlag, 1987.

[10] J.C. Geromel, P.L.D. Peres and S.R. Souza: Mixed $H_{2} / H_{\infty}$ control for continuous-time linear systems. Proc. 31st Conf. Decision and Control, Tuscon, AZ, USA, (1992), 3717-3722.

[11] E.N. Goncalves, R.M. Palhares and R.H.C. Takahashi: Multiobjective optimization applied to robust $\mathrm{H}_{2} / \mathrm{H}_{\infty}$ state-feedback control synthesis. Proc. 2004 American Control Conference, Boston, MA, USA, (2004), 4619-4624.

[12] J. KAUtsky, N. K. Nichols and P. VAN Dooren: Robust pole assignment in linear state feedback. Int. J. Control, 41(5), (1985), 1129-1155.

[13] P.P. Khargonekar and M.A. Rotea: Mixed $\mathrm{H}_{2} / \mathrm{H}_{\infty}$ control. A Convex optimization approach. IEEE Tran. Automatic Control, 36(7), (1991), 824-831. 
[14] D. KROKAVEC and A. FILASOVÁ: LMI constraints on system eigenvalues placement in dynamic output control design. Proc. IEEE Multi-conference on Systems and Control MSC '15, Sydney, Australia, (2015), 1749-1754.

[15] D. Krokavec and A. Filasová: On enhanced mixed $H_{2} / H_{\infty}$ design conditions for control of linear time-invariant systems. Proc. 17th Int. Carpathian Control Conference ICCC 2017, Tatranská Lomnica, Slovakia, (2016), 384-389.

[16] B.P. LAmPe, M. OBRASzo and E.N. RosenwaSser: $H_{2}$-norm computation for stable linear continuous-time periodic systems. Archives of Control Sciences, 14(2), (2004), 147-160.

[17] J. LAN and R.J. PATTON: Integrated design of robust fault estimation and faulttolerant control for linear systems. Proc. 54th Conf. Decision and Control, Osaka, Japan, (2015), 5105-5110.

[18] F.R. Lopez-Estrada, J.C. Ponsart, D. Theilliol, C.M. AstorgaZARAGOZA and J.L. CAMAS-ANZUETO: Robust sensor fault estimation for descriptor-LPV systems with unmeasurable gain scheduling functions. Application to an anaerobic bioreactor. Int. J. Applied Mathematics and Computer Science, 25(2), (2015), 233-244.

[19] M. Meisami-Azad, J. Mohammadpour and K.M. Grigoriadis: Upper bound mixed $H_{2} / H_{\infty}$ control and integrated design for collocated structural systems. Proc. 2009 American Control Conference, St. Louis, MO, USA, (2009), 4563-4568.

[20] K. NonAmi and S. Sivrioglu: Active vibration control using LMI-based mixed $\mathrm{H}_{2} / \mathrm{H}_{\infty}$ state and output feedback control with nonlinearity. Proc. 35th Conf. Decision and Control, Kobe, Japan, (1996), 161-166.

[21] Y.V. ORlov and L.T. Aguilar: Advanced $\mathrm{H}_{\infty}$ Control. Towards Nonsmooth Theory and Applications, New York, Springer Science, 2014.

[22] N. OUCIEF, M. TADJINE and S. LABIOD: Adaptive observer-based fault estimation for a class of Lipschitz nonlinear systems. Archives of Control Sciences, 26(2), (2016), 245-259.

[23] M.A. Rotea and P.P. KhargonekaR: $\mathrm{H}_{2}$-optimal control with an $\mathrm{H}_{\infty}$ constraint. The state feedback case. Automatica, 27(2), (1991), 307-316.

[24] C. SChERER: Mixed $\mathrm{H}_{2} / \mathrm{H}_{\infty}$ control. Trends in Control. A European Perspective, A. Isidori (Ed.), Berlin, Springer-Verlag, (1995), 173-216.

[25] F. Shi and R.J. PATtON: Simultaneous state and fault estimation for descriptor systems using an augmented PD observer. Prepr. 19th IFAC World Congress, Cape Town, South Africa, (2014), 8006-8011. 
[26] F. Shi and R.J. PATton: Fault estimation and active fault tolerant control for linear parameter varying descriptor systems. Int. J. Robust and Nonlinear Control, 25(5), (2015), 689-706.

[27] D. TAN and R.J. PATtON: Integrated fault estimation and fault tolerant control. A joint design. IFAC-PapersOnLine, 48(21), (2015), 517-522.

[28] H. WANG and G.H. YANG: Integrated fault detection and control for LPV systems. Int. J. Robust and Nonlinear Control, 19(3), (2009), 341-363.

[29] Q.G. WANG, Z. Ye, W.J. CAI, and C.C. HANG: PID Control for Multivariable Processes, Berlin, Springer-Verlag, 2008. 